\title{
A Mindfulness-Based Cognitive Therapy (MBCT) Intervention to Improve Resilience and Mitigate Symptoms of Burnout Syndrome in Critical Care Nurses: Results of a Randomized Trial
}

\author{
Meredith Mealer1, Debra Boeldt ${ }^{2}$, Kathryn Cochran1, Rachel Hodapp ${ }^{3}$, Jeri Forster ${ }^{4}$, \\ David Conrad5, Barbara O. Rothbaum ${ }^{6}$, Sona Dimidjian' ${ }^{2}$, Marc Moss ${ }^{3 *}$
}

${ }^{1}$ Department of Physical Medicine and Rehabilitation, University of Colorado School of Medicine, Aurora, CO, USA

${ }^{2}$ Department of Psychology and Neuroscience, University of Colorado Boulder, Boulder, CO, USA

${ }^{3}$ Division of Pulmonary Sciences and Critical Care Medicine, University of Colorado School of Medicine, Aurora, CO, USA

${ }^{4}$ Department of Biostatistics and Informatics, Colorado School of Public Health, University of Colorado Denver, Aurora, CO, USA

${ }^{5}$ Department of Pediatrics, University of Colorado School of Medicine, Aurora, CO, USA

${ }^{6}$ Department of Psychiatry, Emory University School of Medicine, Atlanta, GA, USA

Email: *Marc.Moss@CUAnschutz.edu

How to cite this paper: Mealer, M., Boeldt, D., Cochran, K., Hodapp, R., Forster, J., Conrad, D., Rothbaum, B.O., Dimidjian, S. and Moss, M. (2021) A Mindfulness-Based Cognitive Therapy (MBCT) Intervention to Improve Resilience and Mitigate Symptoms of Burnout Syndrome in Critical Care Nurses: Results of a Randomized Trial. Open Journal of Nursing, 11, 653-667. https://doi.org/10.4236/ojn.2021.118055

Received: July 13, 2021

Accepted: August 13, 2021

Published: August 16, 2021

Copyright $\odot 2021$ by author(s) and Scientific Research Publishing Inc. This work is licensed under the Creative Commons Attribution International License (CC BY 4.0).

http://creativecommons.org/licenses/by/4.0/ (c) (i) Open Access

\begin{abstract}
Objective: Critical care nurses work in a challenging intensive care (ICU) environment that results in work-related psychological distress. Our objective was to pilot an in-person or virtual mindfulness-based cognitive therapy (MBCT) program enhanced resilience and a similarly designed attention control group. Methods: We randomized ICU nurses with symptoms of burnout syndrome and decreased resilience to an $\mathrm{MBCT}$ program or a similarly formatted book club control. Our primary outcome was change in resilience as measured by the Connor-Davidson Resilience Scale (CD-RISC). Results: Onehundred one nurses completed study-related procedures. Overall, $70 \%$ had baseline symptoms of anxiety and $26 \%$ had symptoms of depression. For the in-person cohorts, there was no statistical difference between intervention and control groups regarding the total number of sessions attended ( 3.85 days \pm 1.4 versus 3.75 days $\pm 0.15 ; \mathrm{p}=0.64)$. Using the Client/Patient Satisfaction Questionnaire-8 (CSQ-8), satisfaction scores were higher in the intervention group for weeks two through four of the program: $p=0.03,0.0003,0.007$ respectively. There was no difference in the change in CD-RISC scores between the two groups (mean difference: treatment $=5.0$, control $=7.0 ; \mathrm{p}=0.30$ ). The online intervention cohort had greater improvements in the change of
\end{abstract}


their median emotional exhaustion burnout scores when compared to the inperson intervention cohorts $(-5[-8$ to -1.5$]$ vs. $2[-5$ to 8$], \mathrm{p}=0.049)$.

Conclusions: We developed a feasible and acceptable in-person and online MBCT-ICU intervention that did not increase resilience scores in ICU nurses when compared to an attention control group. These results could help guide the proper design of larger trials to determine the efficacy of other resilience interventions.

\section{Keywords}

Burnout Syndrome, Mindfulness, Cognitive Behavioral Therapy, Critical Care, Nursing

\section{Introduction}

Intensive care unit (ICU) nurses have difficult jobs related to repetitive traumatic direct patient care experiences [1]. The cumulative exposure to these work-related stresses may cause significant psychological distress. The risk of psychological distress is high in ICU nurses with increased rates of burnout syndrome (BOS), posttraumatic stress disorder (PTSD), depression and anxiety [2] [3].

These stressful experiences are intrinsic to the critical care environment including coping with end-of-life issues, patient deaths, and performing cardiopulmonary resuscitation. While there are organizationally focused interventions that can be beneficial to support clinician wellbeing such as debriefings and pauses, individually focused resilience building activities may also be beneficial. Resilience is a psychological construct that buffers the impact of stress and trauma, resulting in a return to baseline functioning without long-term consequences [4] [5] [6]. There are resilience psychological characteristics that are modifiable, and therefore resilience can be learned [4]. One of these characteristics is cognitive flexibility or the ability to reframe a perspective or experience related to a stressful situation [7]. Cognitive flexibility can be enhanced by mindfulness and meditative training and is associated with improved wellbeing [8]. Higher levels of resilience in ICU nurses are associated with less symptoms of BOS or PTSD [9].

There are currently no established and accepted individually focused resilience building activities for ICU nurses. Mindfulness Based Cognitive Therapy (MBCT) integrates Mindfulness Based Stress Reduction and Cognitive Behavioral Therapy and helps individuals bring awareness towards negative feelings and thoughts [10] [11]. MBCT teaches individuals to think differently about troubling experiences and can building resilience [10] [11] [12] [13] [14]. In an 8-week in-person group format, MBCT has been evaluated for patients with anxiety, stress, and PTSD [10]. Additionally, a digital MBCT format has been developed that could increase access to the intervention [15]. However, there are no studies examining the effect of MBCT on resilience, psychological distress, or burnout in ICU 
nurses.

The goal of this randomized trial was to determine whether an in-person MBCT resilience program was acceptable and feasible to ICU nurses, identify recruitment and randomization challenges for future studies, and determine whether the intervention improved resilience when compared to an attention control group. We also wanted to explore subgroups that may have improved responsiveness to the $\mathrm{MBCT}$ intervention and examine the ability to conduct the MBCT program with a web-based platform.

\section{Methods}

ICU nurses were enrolled from Denver metropolitan area hospitals from July 2017 until April 2018. Eligible nurses were recruited formal announcements at staff meetings, posting of flyers in the hospital, and word of mouth. The study inclusion criteria included: nurses who are employed by a hospital and working in the ICU for at least 20 hours per week that manifest decreased resilience defined as a score of $<82$ on the Connor-Davidson Resilience Scale (CD-RISC) and had positive symptoms of BOS in at least one dimension, using the Maslach Burnout Inventory (MBI): $>17$ emotional exhaustion score, $>7$ depersonalization score, or <31 personal accomplishment score [16] [17] [18]. The completion of the above scales for inclusion into the study were performed under a waiver of consent and HIPAA authorization. Our exclusion criterion was part-time or per diem nurses working less than 20 hours per week at the bedside. Informed consent was obtained from all study subjects and the voluntary nature of the study as well as the ability to withdraw from the study was explained to all participants. A computerized randomization table was used to generate treatment assignment in a 1:1 allocation, which was performed by the trial project manager.

Patient and Public Involvement: With stakeholder engagement, we optimized the MBCT program. ICU nurses, ICU based managers, and hospital administrators participated in focus groups to determine components of the program including session scheduling, course integration into care models, an acceptable control group, the amount of reimbursement, and identification of important outcomes [19]. Through word of mouth, ICU nurses helped with recruitment of subjects. We did not assess the burden of the intervention on the ICU nurses. Results will be disseminated through presentations at national nursing meetings.

Ethics Statement: This study was approved by the Colorado Multiple Institutional Review Board (COMIRB\# 17-0279). All participants gave their informed consent before their involvement in any research related activities. Any revisions of the MBCT or control interventions were reviewed and approved by COMIRB prior to implementation. Data from this study will be made available upon request. At the time of enrollment, we collected individual participant and workrelated demographics. We also collected responses to the following questionnaires listed below

The Connor-Davidson Resilience Scale (CD-RISC) is a 25 item self-report 
questionnaire developed to measure clinical treatment response and to assess individual levels of resilience [17]. Total score ranges from 0 - 100 with scores $\geq 82$ reflecting average levels of resilience and $\geq 92$ reflecting high levels of resilience [20].

Maslach Burnout Inventory (MBI)-HS is a 22-item self-report questionnaire that measures the three dimensions of burnout syndrome (emotional exhaustion (EE), depersonalization (DP) and personal accomplishment (PA)) on a 7-point Likert scale [16] [21]. The EE scale identifies individuals who are overextended or emotionally exhausted at work and consists of 9 items. Individuals who have an impersonal response towards their patients is measured on the DP scale, which includes 5 items. The PA scale assesses levels of accomplishment and success related to work and includes 8 items [22].

The Posttraumatic Diagnostic Scale (PDS) is a self-report tool that measures PTSD symptom severity and meets all diagnostic criteria for a PTSD diagnosis. The PDS is highly correlated with the gold standard clinician-administered PTSD scale (CAPS) [18] [23] [24]. Symptoms are scored using a 4-point scale and diagnostic criteria are met if there is an identifiable traumatic event, one episode of re-experiencing the trauma, three symptoms of avoidant behavior, and two symptoms of hyperarousal. Symptom severity can be calculated by summing the scores of the three sections related to re-experiencing, avoidance and hyperarousal symptoms. For this study, nurse participants were asked to focus on a work-related trauma and symptoms related to that trauma.

The Hospital Anxiety and Depression Scale (HADS) is a self-report measure that includes 14 questions: 7 questions addressing symptoms of depression and 7 questions addressing symptoms of anxiety. A score that indicates a suggestive diagnosis of anxiety or depression is $>8$ on each of the subscales [1] [25] [26].

Client/Patient Satisfaction Questionnaire-8 (CSQ-8) is a short measure of satisfaction that includes eight items rated on a 4-point Likert scale. Satisfaction is calculated by summing the scores of each question with higher scores indicating higher levels of satisfaction [27]. The CSQ-8 has demonstrated good validity and reliability [28]. The CSQ-8 was used to determine satisfaction with the MBCT and control interventions.

Due to the sensitive nature of the questionnaires and the intervention, we used a third-party honest broker to ensure that participant responses remained anonymous and all data was de-identified. The honest broker was provided by the University of Colorado Clinical Translational Science Award research core, and the individual was not part of the research team nor in the same department as the research team. Study participants entered their demographic information and survey responses into an electronic database using study identification numbers to ensure that the study team remained blinded to personally identifiable information. The honest broker then linked the study identification numbers with personally identifiable information and longitudinal data collection. Subsequently, they de-identified the data and transferred this data electronically to 
the study team.

\section{Primary Outcome:}

Change in CD-RISC scores: The primary outcome was the pre-post changes in the CD-RISC scores in intervention and control subjects.

\section{Secondary Outcomes:}

Acceptability: Overall satisfaction scores were calculated to measure acceptability, using the CSQ-8 [27]. Scores can range from 8 to 32 , and higher scores indicate greater levels of satisfaction. The CSQ- 8 was administered weekly after each session.

Adherence: Our research personnel recorded study participant's attendance at each of the sessions and completion of assigned daily practices. Through our honest broker system, study participants completed REDCap diary cards and recorded their weekly participation in their out of class activities. Adherence was calculated from these two sources of information.

MBCT-ICU Intervention: The intervention was adapted using input from focus groups with critical care nurses and administrators. Similar to the structure of standard MBCT courses, the MBCT-ICU program consisted of 16 hours of MBCT training. All sessions followed a consistent format. Each session began with a meditation to facilitate transition from the "doing" mode to "being" mode, focused on non-judgmental awareness of the present moment. This practice was followed by a process of inquiry during which the facilitator guided discussion and self-reflection about experiences with the in-class mindfulness practice and the daily home practice assignments. New material was introduced through experiential exercises followed by group discussion. The first half of the program focused on developing a foundation of mindfulness skills. The second half of the program emphasized the application of mindfulness skills to the context of work and focused on developing skills in responding flexibly and intentionally, rather than reactively, at times of stress at work. Acceptance and non-judgmental awareness as skillful steps in dealing with one's work environment were explicitly explored. The nurse participants were asked to practice mindfulness daily and complete homework assignments to reinforce behaviors learned during class time. The complete intervention protocol is included in the supplementary material.

Control group: Our control protocol was an attention comparison group in the format of a book club. A novel was chosen by the study team and each cohort used that novel (A Man Called Ove by Fredrik Backman). This novel that did not include events that may trigger work-related experiences. The group met for the same number of sessions and duration as the intervention group. During each session, participants had free reading time, open discussion about the book, and activities that fostered group communication such as playing board games. The instructor discouraged discussions regarding work, and no mindfulness skills were discussed or implemented. Homework was assigned reading time that was matched to the amount and duration of homework as the intervention group. 
Phase 1: We initially conducted a pre-pilot cohort with the following goals: to enhance the feasibility of MBCT-ICU and control interventions, refine the logistics of the programs, and initially evaluate treatment and instructor fidelity. Participants attended a total of 8 two-hour weekly sessions. Each MBCT-ICU and control session were led by two instructors. For the MBCT-ICU group, both instructors were formally trained in $\mathrm{MBCT}$ and one was also an experienced ICU nurse. For the control group, one instructor was a licensed clinical social worker and the other was an experienced ICU nurse. Group qualitative interviews were conducted with participants at the conclusion of the program.

Phase 2: Based on feedback identified from the study participants in Phase 1, we adjusted the intervention and control protocol to become four 4-hour sessions and consolidated the meditation home practice to remove any duplicates based on the prior week. In addition, we only had one instructor for each of the intervention and control sessions. Finally, we conducted the control and intervention sessions on the same day and at the same time of day. This change allowed randomization to occur a few days before the course with the hope to increase engagement and decrease the rate of drop out prior to the commencement of the sessions.

Phase 3: The intervention and control programs were delivered in a webbased format to provide more flexibility in completing the program. We used a web-based adaptation of MBCT called Mindful Mood Balance (MMB). MMB is an 8-week program that was modified to fit the 4 -week format introduced in phase 2. Introductory emails were completed with the participants, at which time they were set up with MMB accounts and questions about the study were answered. The instructor offered to schedule an optional introduction call to answer questions and discuss the program and process. Participants were instructed to complete two MMB sessions a week and the homework was built into the program. In addition, a weekly group one-hour video conference session was conducted with a trained MBCT instructor to discuss how the MMB skills apply to burnout syndrome in the ICU. Based on availability, participants chose from several meeting times during the week. The video conference session started with a meditation and brief inquiry. Participants discussed their home practice relative to working in the ICU. Prior to each video conference, the MBCT instructor sent a reminder email that contained a link and clarified the sessions that should be completed. Throughout the 4-week program, the instructor responded to technical questions about the program and about the progression of the program or home practice. The MMB sessions included: mindfulness training, the body scan, the breath, exploring the landscape of burnout syndrome, facing difficulties, the concept that thoughts are not facts, learning how to build your plan of action, and supporting your practice in the world introduction.

The online book club included 4 weekly sessions. Web-based book club participants were asked to do home reading, respond to discussion questions, and 
attend one group video conference session per week that last an hour. The group video conference sessions were offered on two different days and participants were given the option to choose the best day for them each week. During the initial session, the book club facilitator met with the group to establish general rules, expectations, engage in ice-breaker exercises and discuss the book chapters. The participants were given the assigned reading schedule (approximately 100 pages each week). Participants kept a daily reading log where they entered the minutes spent reading. Like the MBCT group, book club participants completed pre/post surveys (before week 1 and after week 4) and filled out weekly homework logs and satisfaction surveys.

Statistical Analysis. All comparisons assumed a two-sided test of hypothesis and a significance level of 0.05. Baseline demographics were compared between groups using two-sample t-tests, chi-square, and Fisher's exact tests, as appropriate. The difference in change on all outcome measures were compared using two-sample t-tests for Phases 2 and 3, as were the weekly CSQ-8 scores. Wilcoxon rank-sum tests were used to assess differences for Phase 1. Linear regression was then used to model the change in each outcome as a function of group as well as baseline demographics that were 1) found to be statistically significantly different between the groups and 2) have a plausible confounding effect on the relationship of interest. Additional post-hoc analyses were performed to examine the differences between virtual and in-person attendance for both the book club and the MBCT program. CSQ-8 scores and change in burnout sub-scores (emotional exhaustion, and depersonalization) were compared within intervention groups and between in-person and virtual delivery modes using a nonparametric Wilcoxon test. Lastly, we ran an exploratory post-hoc analysis which compared the change in emotional exhaustion between intervention groups based on their baseline CD-RISC scores. We examined three additional and more conservative cutoff values for the baseline CD-RISC $(75,70$, and 65$)$. These comparisons were made using nonparametric Wilcoxon tests. Previous studies suggest that clinically significant changes in CD-RISC scores range from 12 - 19 points with an approximate standard deviation of 20 points [17]. With a total enrollment of 120 subjects, we will be able to detect a difference of 12 points in CD-RISC score between the control and intervention groups with $90 \%$ power (assuming a 2 sided significance level (alpha) of 0.05 and an equal variance $t$ test. There were no planned interim analyses.

\section{Results}

During all three phases of the study, 233 ICU nurses expressed interest in participating in the trial. Of those interested, 24 did not complete the screening process and 48 were not included due to either not meeting the inclusion criteria or they met the exclusion criterion. Of the 161 eligible nurses, 139 consented to the study (consent rate of $86 \%$ ). Subsequently, eight nurses withdrew prior to randomization. Overall, 131 participants were randomized with 66 rando- 
mized to the control arm (book club) and 65 randomized to the intervention arm (MBCT-ICU). A total of 30 nurses (22.9\%; 95\% point estimate confidence interval $=15.7 \%-30.1 \%)$ withdrew from the study. Twenty-five withdrew after randomization and prior to the start of the intervention, 8 from the control arm and 17 from the intervention arm. Five subjects withdrew during the treatment period, four from the control arm and one from the intervention arm (see Figure 1). One hundred one nurses completed a portion or all study-related procedures. Overall, $70 \%$ of the participants had symptoms of anxiety and $26 \%$ had symptoms of depression at baseline. Of the participants with symptoms of anxiety, $14 \%(10 / 74)$ were taking medications for their symptoms. Of the participants with symptoms of depression, 50\% (14/28) were taking medications for their symptoms. Table 1 includes the demographic characteristics of the enrolled ICU nurse subjects.

\section{Phase 1:}

Overall, 13 individuals who participated in Phase 1 of the study: 5 in the MBCT-ICU and 8 in the control group. There was a significant difference between groups in the total number of sessions attended (MBCT-ICU group $6.8 \pm$ 1.0 compared to the control group $5.0 \pm 0.9, \mathrm{p}$ value $=0.009)$. On average, the

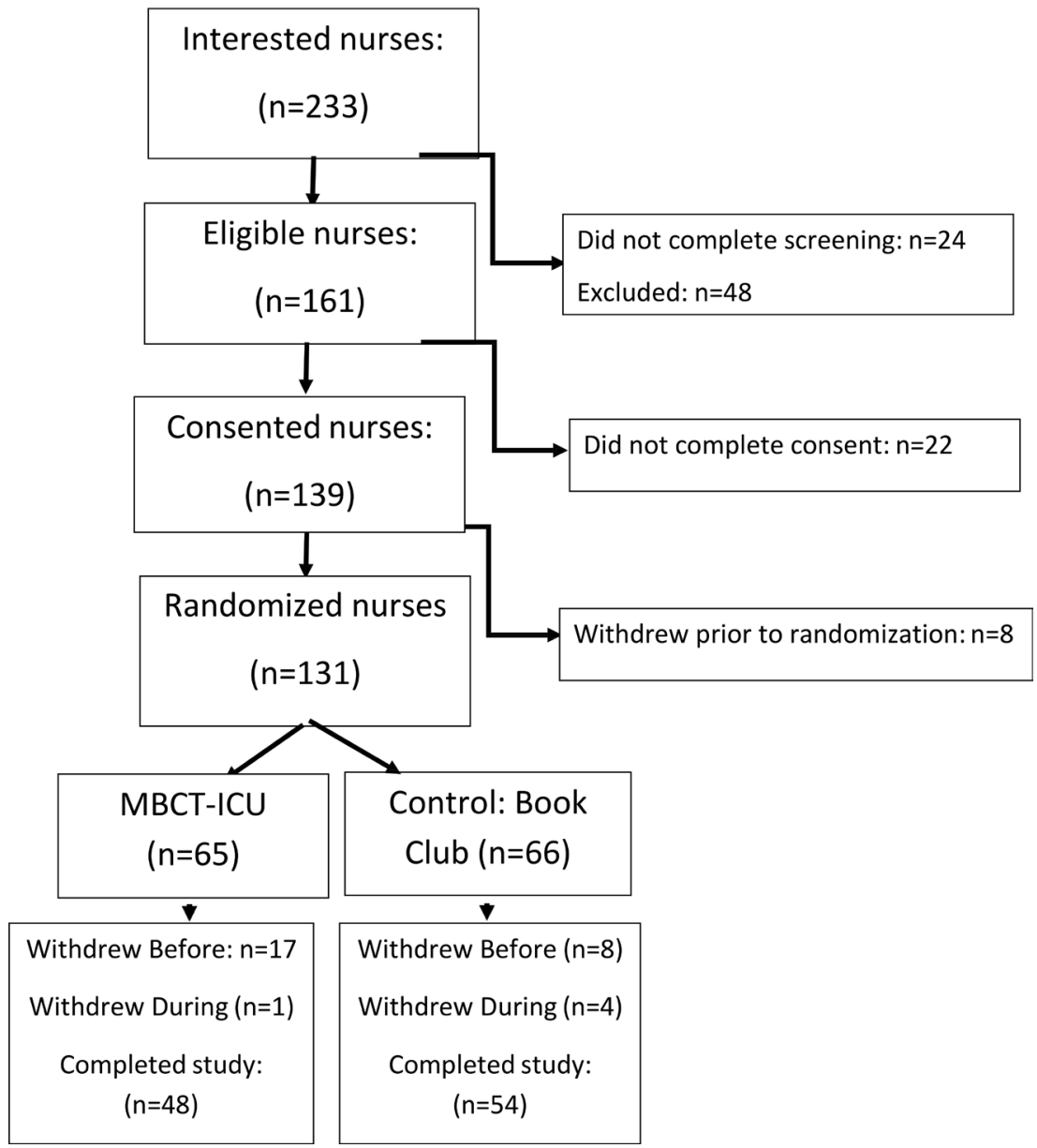

Figure 1. Enrollment, randomization, and follow-up. 
Table 1. Baseline demographics.

\begin{tabular}{|c|c|c|c|}
\hline Variable & $\begin{array}{c}\text { Treatment (MBCT) } \\
\qquad \mathbf{N}=\mathbf{4 8}\end{array}$ & $\begin{array}{l}\text { Control (Book Club) } \\
\qquad \mathrm{N}=58\end{array}$ & p-value \\
\hline \multicolumn{4}{|l|}{ Gender } \\
\hline$\%$ female $(n)$ & $94 \%(45 / 48)$ & $93 \%(54 / 58)$ & 0.90 \\
\hline Age (mean/SD) & $33.0 \pm 6.5$ & $32.4 \pm 7.4$ & 0.73 \\
\hline \multicolumn{4}{|l|}{ Race } \\
\hline$\%$ Caucasian $(\mathrm{n})$ & $92 \%(44 / 48)$ & $95 \%(55 / 58)$ & 0.07 \\
\hline \multicolumn{4}{|l|}{ Ethnicity } \\
\hline \% Hispanic (n) & $2 \%(1 / 48)$ & $3 \%(2 / 58)$ & 0.67 \\
\hline \multicolumn{4}{|l|}{ Marital Status } \\
\hline$\%$ Married (n) & $54 \%(26 / 48)$ & $47 \%(27 / 58)$ & 0.30 \\
\hline$\%$ with Children (n) & $25 \%(12 / 48)$ & $22 \%(13 / 58)$ & 0.80 \\
\hline \multicolumn{4}{|l|}{ Highest Nursing Degree } \\
\hline$\% \operatorname{BSN}(\mathrm{n})$ & $92 \%(44 / 48)$ & $92(\% 53 / 58)$ & 0.57 \\
\hline Years Practicing as a nurse (mean/SD) & $6.5 \pm 1.0$ & $6.8 \pm 0.9$ & 0.80 \\
\hline Years Practicing ICU (mean/SD) & $5.2 \pm 5.9$ & $5.3 \pm 0.8$ & 0.85 \\
\hline \multicolumn{4}{|l|}{ ICU Type } \\
\hline$\% \operatorname{MICU}(\mathrm{n})$ & $25 \%(12 / 48)$ & $19 \%(11 / 58)$ & 0.23 \\
\hline \multicolumn{4}{|l|}{ Patient/Nurse Ratio } \\
\hline$\% 2: 1(n)$ & $75 \%(36 / 48)$ & $78 \%(45 / 58)$ & 0.31 \\
\hline \multicolumn{4}{|l|}{ Primary Shift } \\
\hline$\%$ Days (n) & $44 \%(21 / 48)$ & $36 \%(21 / 58)$ & 0.72 \\
\hline \multicolumn{4}{|l|}{ Medications } \\
\hline Anxiety (\% yes) (n) & $4 \%(2 / 48)$ & $14 \%(8 / 58)$ & 0.08 \\
\hline Depression (\% yes) (n) & $6 \%(3 / 48)$ & $19 \%(11 / 58)$ & $0.05^{*}$ \\
\hline \multicolumn{4}{|l|}{ Regular Exercise } \\
\hline$\%$ yes $(n)$ & $94 \%(45 / 48)$ & $90 \%(52 / 58)$ & 0.45 \\
\hline \multicolumn{4}{|l|}{ Practice Mindfulness } \\
\hline$\%$ yes $(n)$ & $8 \%(4 / 48)$ & $24 \%(14 / 58)$ & $0.03^{*}$ \\
\hline \multicolumn{4}{|l|}{ Current Therapy/Counseling } \\
\hline$\%$ yes $(\mathrm{n})$ & $21 \%(10 / 48)$ & $21 \%(12 / 58)$ & 0.98 \\
\hline \multicolumn{4}{|l|}{ HADS-Anxiety } \\
\hline$\%$ positive $(\mathrm{n})$ & $60 \%(29 / 48)$ & $78 \%(45 / 58)$ & $0.04^{*}$ \\
\hline \multicolumn{4}{|l|}{ HADS-Depression } \\
\hline \multicolumn{4}{|l|}{ MBI-Emotional Exhaustion } \\
\hline$\%$ positive $(\mathrm{n})$ & $97 \%(47 / 48)$ & $79 \%(46 / 58)$ & $0.004^{*}$ \\
\hline \multicolumn{4}{|l|}{ MBI-Depersonalization } \\
\hline$\%$ positive $(\mathrm{n})$ & $77 \%(37 / 48)$ & $71 \%(41 / 58)$ & 0.46 \\
\hline \multicolumn{4}{|l|}{ MBI-Reduced Personal Accomplishment } \\
\hline$\%$ positive $(\mathrm{n})$ & $58 \%(28 / 48)$ & $71 \%(41 / 48)$ & 0.18 \\
\hline
\end{tabular}

MBCT-ICU group completed $686 \pm 513$ minutes of home practice and the control group completed $406 \pm 227$ minutes, $\mathrm{p}=0.20$. There was also no significant difference between the MBCT-ICU group and the control group in the CSQ-8 satisfaction scores (See Table 2). 
Table 2. Satisfaction (Phase 1 Cohort).

\begin{tabular}{ccccrc}
\hline \multirow{2}{*}{ Measure: CSQ-8 } & \multicolumn{2}{c}{ Treatment } & \multicolumn{2}{c}{ Control } & \multirow{2}{*}{ p-value } \\
\cline { 2 - 4 } & $\mathrm{N}$ & Mean (SD) & $\mathrm{N}$ & Mean (SD) & \\
\hline Week 1 & 5 & $25.0(2.12)$ & 6 & $23.8(1.33)$ & 0.29 \\
Week 2 & 4 & $24.5(3.32)$ & 6 & $24.5(2.95)$ & 1.00 \\
Week 3 & 5 & $25.6(3.21)$ & 6 & $22.7(2.66)$ & 0.13 \\
Week 4 & 3 & $22.3(2.89)$ & 3 & $26.70(4.61)$ & 0.24 \\
Week 5 & 3 & $25.7(2.10)$ & 2 & $27.5(6.40)$ & 0.65 \\
Week 6 & 4 & $26.8(3.77)$ & 5 & $25.8(6.34)$ & 0.80 \\
Week 7 & 3 & $28.7(4.16)$ & 0 & $* *$ & $* *$ \\
Week 8 & 4 & $27.5(3.11)$ & 3 & $23.0(7.00)$ & 0.30 \\
\hline
\end{tabular}

\section{Phase 2 and 3: Attendance and Acceptability.}

There was no statistical difference between groups regarding the total number of sessions attended (MBCT group 3.85 days \pm 1.4 vs. the control group 3.75 days $\pm 0.15 ; \mathrm{p}=0.64)$. On average, the MBCT-ICU group completed $438 \pm 237$ minutes of home practice and the control group completed $474 \pm 340$ minutes. There was no statistical difference in home practice between the two groups, $\mathrm{p}=$ 0.53 . The week 1 CSQ scores were not significantly different between groups (MBCT-ICU $26.2 \pm 3.2$ vs. control $25.5 \pm 3.1 ; \mathrm{p}=0.27$ ). However, weeks $2-4$ showed significantly increased satisfaction in the MBCT-ICU group compared with the control group (Table 3 and Figure 2).

In unadjusted comparisons across the outcomes of interest (Table 4), none of the group differences achieved statistical significance. Specifically, there was no difference in the change in CD-RISC scores between the two groups (mean difference: treatment $=5.0$, control $=7.0 ; p=0.30$ ). When adjusting for the four variables found to be different between groups at baseline (\% of participants taking medications for depression at baseline, \% practicing mindfulness at baseline, \% suggestive of having anxiety at baseline and \% suggestive of emotional exhaustion at baseline), the differences in the outcome variables remained nonsignificant. There were no reported adverse events.

Analyses based on in-person versus virtual delivery:

When comparing the phase 2 (in-person) and phase 3 (virtual) participants who were randomized to the MBCT intervention, in-person cohort had higher CSQ-8 scores at week 1 and 4 when compared to the virtual cohort (week 1: 28 [25 - 29] vs. 24.5 [24 - 27], $\mathrm{p}=0.04$; week $2: 28$ [26 - 30] vs. 24 [24 - 27], $\mathrm{p}=0.10$; week 3: 29 [28 - 32] vs. 27 [25 - 28], p = 0.05; week 4: 31 [28 - 32] vs. 25 [23 - 27], $\mathrm{p}=0.003)$. However, the phase 3 virtual cohort had greater improvements in the change in their median emotional exhaustion scores when compared to the phase 2 in-person group ( -5 [ -8 to -1.5$]$ vs. 2 [ -5 to 8$], \mathrm{p}=0.049)$.

Analyses based on CD-RISC scores:

In our post-hoc analysis, we identified that for the 39 participants with lower 
Table 3. Satisfaction (Phase $2 \&$ Phase 3 Cohorts).

\begin{tabular}{cccccc}
\hline \multirow{2}{*}{ Measure: CSQ-8 } & \multicolumn{2}{c}{ Treatment } & \multicolumn{2}{c}{ Control } & \multirow{2}{*}{ p-value } \\
\cline { 2 - 4 } & $\mathrm{N}$ & Mean (SD) & $\mathrm{N}$ & Mean (SD) & \\
\hline Week 1 & 46 & $26.2(3.2)$ & 49 & $25.5(3.1)$ & 0.27 \\
Week 2 & 40 & $27.0(3.0)$ & 48 & $25.4(3.4)$ & $\mathbf{0 . 0 3 ^ { * }}$ \\
Week 3 & 40 & $28.2(3.4)$ & 45 & $24.9(4.4)$ & $\mathbf{0 . 0 0 0 3 ^ { * }}$ \\
Week 4 & 37 & $28.2(3.8)$ & 42 & $25.5(4.8)$ & $\mathbf{0 . 0 0 7}^{*}$ \\
\hline
\end{tabular}

Table 4. Pre-, post- and mean differences.

\begin{tabular}{cccccccc}
\hline \multirow{2}{*}{ Measure } & \multicolumn{2}{c}{ Pre-Mean (SD) } & \multicolumn{2}{c}{ Post-Mean (SD) } & \multicolumn{2}{c}{ Mean Difference } & \multirow{2}{*}{ p-value } \\
\cline { 2 - 6 } & Treatment & Control & Treatment & Control & Treatment & Control & \\
\hline CD-RISC & $67.6 \pm 7.9$ & $66.9 \pm 9.1$ & $72.3 \pm 9.4$ & $73.6 \pm 10.3$ & 5.0 & 7.0 & 0.30 \\
$\begin{array}{c}\text { MBI Emotional } \\
\text { Exhaustion }\end{array}$ & $29.2 \pm 9.1$ & $26.4 \pm 9.8$ & $29.0 \pm 9.1$ & $24.9 \pm 10.2$ & -0.49 & -1.0 & 0.78 \\
$\begin{array}{c}\text { MBI } \\
\text { Depersonalization }\end{array}$ & $11.8 \pm 5.7$ & $10.8 \pm 6.0$ & $12.7 \pm 5.9$ & $10.5 \pm 6.5$ & 1.1 & 0.2 & 0.35 \\
$\begin{array}{c}\text { MBI } \\
\text { Reduced Personal } \\
\text { Accomplishment }\end{array}$ & $30.8 \pm 5.7$ & $29.9 \pm 6.3$ & $34.0 \pm 6.5$ & $34.1 \pm 7.1$ & 3.4 & 4.1 & 0.64 \\
HADS-Anxiety & $9.0 \pm 3.4$ & $9.6 \pm 3.3$ & $8.6 \pm 3.2$ & $8.0 \pm 3.2$ & -0.4 & -1.4 & 0.08 \\
HADS-Depression & $5.8 \pm 3.9$ & $5.7 \pm 4.0$ & $4.7 \pm 4.1$ & $4.5 \pm 4.2$ & -1.0 & -0.8 & 0.67 \\
PDS-Intrusion & $4.3 \pm 2.8$ & $3.9 \pm 3.1$ & $4.5 \pm 3.2$ & $3.7 \pm 3.2$ & 0.36 & 0.06 & 0.67 \\
$\begin{array}{c}\text { PDS-Avoidance } \\
\text { PDS-Arousal }\end{array}$ & $1.9 \pm 1.5$ & $2.0 \pm 1.6$ & $2.0 \pm 1.7$ & $1.5 \pm 1.4$ & 0.1 & -0.3 & 0.17 \\
\hline
\end{tabular}

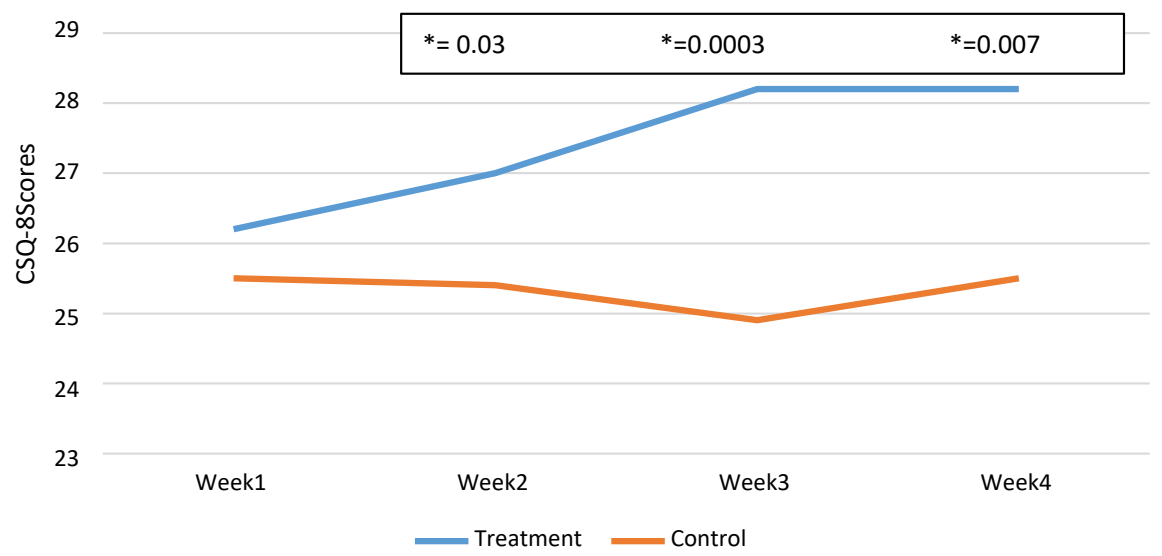

Figure 2. Satisfaction scores for Phase $2 \&$ Phase 3 participants.

CD-RISC (defined as $\leq 65$ ) the MBCT-ICU group showed a trend towards improved changes in emotional exhaustion compared to the book club (MBCT: -3 [ -8.25 to 1.75$]$ and book club: 4 [ -4.75 to 5.5$], \mathrm{p}=0.18$ ). There was no difference when a pre-CD-RISC cutoff of 70 or 75 were used. 


\section{Discussion}

In this study, we developed, and tested a modified MBCT resilience intervention for critical care nurses. This is the first study to adapt an MBCT intervention for ICU nurses. The modified MBCT was both feasible to conduct and acceptable to the participants based on their satisfaction scores. Our prior work had reported focus group qualitative evidence related to potential barriers associated with program adherence [19]. However, there was no difference in the change in resiliency scores between the two groups. The Phase 3 intervention demonstrated that the adoption of a shorter, hybrid online MBCT program was feasible. Web-based MBCT is a cost-effective, time-efficient option of delivering MBCT to critical care nursing professionals and may increase access to those nurses who do not work in metropolitan areas and enhance adherence to treatment. In addition, a post-hoc analysis demonstrated that the MBCT intervention may be more effective with the enrollment of nurses with lower baseline resiliency scores. Finally, we learned that participant drop out is common in this cohort of ICU nurses. This information will be important in determining the proper sample size for future studies.

In the pre-pilot group (phase 1), we learned that an 8-week MBCT intervention was too long for critical care nurses and resulted in participant dropout. Modifying the 8-week MBCT intervention to a 4-week intervention improved retention of subjects. Additionally, we learned that we should target a $20 \%-25 \%$ increase in the number of participants needed for enrollment to account for dropouts during the study and prior to the study being initiated. Finally, based on satisfaction scores, participants found the in-person MBCT intervention acceptable.

There were no statistically significant reductions in resilience, PTSD, anxiety or depression symptom severity scores. However, it is possible the survey instruments may not be as responsive to change as anticipated in this patient population. In addition, we maybe did not test these outcomes at the proper time after completion of the intervention. Therefore, the MBCT-ICU intervention may be worth exploring in a sufficiently powered clinical trial with different outcome variables assessed at different time points. Interestingly, while there was a positive signal for improved emotional exhaustion scores and personal accomplishment scores on the MBI-HS burnout measure, depersonalization scores were significantly higher in the MBCT group compared with the control group. Depersonalization is a clinical providers' callous or dehumanized perception of their patients [22]. However, it is also assumed that depersonalization is positively correlated with emotional exhaustion [22]. Our results may suggest a negative correlation. This could potentially be explained by the potential lack of validity of the MBI-HS in ICU nurses. Recent evidence suggests that the MBI-HS is not a 3-dimension measure of emotional exhaustion, depersonalization and personal accomplishment. Instead, an exploratory and confirmatory factor analysis in critical care nurses suggests a 2 -dimension measure with roughly $40 \%$ of the 
depersonalization questions removed due to poor factor loadings and non-normality of distribution [29].

A strength of our study was that the program was adapted based on the needs of the ICU nurses and the use of an attention control group. We adapted both the program length and format, which improved engagement and attendance. The control was well matched to the intervention group for number of sessions and duration of each session. Studies that use controls with no exposures are more likely to report a treatment effect. However, this observed treatment effect may be due to the communal nature of the study delivery as opposed to the actual intervention.

Our study is not without limitations. This was a voluntary trial that required informed consent for participation. There may be an associated self-selection bias and overrepresentation of ICU nurses who had a positive perception of mindfulness practices. Second, due to drop out of $22.9 \%$ of participants, we did not reach our predetermined sample size of 120 patients. Therefore, our inability to determine a beneficial effect of the intervention on our outcome variable (change in CD-RISC score) could be due to an underpowered study. However, if anything the attention control group had a greater change in CD-RISC scores when compared to the intervention. Finally, we used self-report surveys to measure resilience, PTSD, BOS, anxiety and depression instead of clinician rating scales.

\section{Conclusion}

In conclusion, we developed a feasible and acceptable MBCT-ICU resilience intervention for critical care nurses. The MBCT intervention was modified based on feedback received by participants in a pilot of the traditional 8-weekly 16hour MBCT program and participants in a 4-weekly 16-hour MBCT program. However, the intervention did not achieve the pre-determined improvement in resilience based on the change in CD-RSIC scores.

\section{Fund}

This research was funded by National Institutes of Health grant number R34AT009181.

\section{Conflicts of Interest}

There are no conflicts of interest, financial disclosures, or competing interests for any of the authors.

\section{References}

[1] Mealer, M.L., Shelton, A., Berg, B., Rothbaum, B. and Moss, M. (2007) Increased Prevalence of Posttraumatic Stress Disorder Symptoms in Critical Care Nurses. American Journal of Respiratory and Critical Care Medicine, 175, 693-697. https://doi.org/10.1164/rccm.200606-735OC

[2] Czaja, A.S., Moss, M. and Mealer, M. (2012) Symptoms of Posttraumatic Stress Disorder among Pediatric Acute Care Nurses. Journal of Pediatric Nursing, 27, $357-$ 
365. https://doi.org/10.1016/j.pedn.2011.04.024

[3] Mealer, M., Burnham, E.L., Goode, C.J., Rothbaum, B. and Moss, M. (2009) The Prevalence and Impact of Post Traumatic Stress Disorder and Burnout Syndrome in Nurses. Depression and Anxiety, 26, 1118-1126. https://doi.org/10.1002/da.20631

[4] Charney, D.S. (2004) Psychobiological Mechanisms of Resilience and Vulnerability: Implications for Successful Adaptation to Extreme Stress. American Journal of Psychiatry, 161, 195-216. https://doi.org/10.1176/appi.ajp.161.2.195

[5] Dyrbye, L. and Shanafelt, T. (2012) Nurturing Resiliency in Medical Trainees. Medical Education, 46, 343. https://doi.org/10.1111/j.1365-2923.2011.04206.x

[6] Howe, A., Smajdor, A. and Stockl, A. (2012) Towards an Understanding of Resilience and Its Relevance to Medical Training. Medical Education, 46, 349-356. https://doi.org/10.1111/j.1365-2923.2011.04188.x

[7] Lacoviello, B. and Charney, D. (2014) Psychosocial Facets of Resilience: Implications for Preventing Posttrauma Psychopathology, Treating Trauma Survivors and Enhancing Community Resilience. European Journal of Psychotraumatology, 5, Article No. 23970. https://doi.org/10.3402/ejpt.v5.23970

[8] Raffone, A. and Srinivasan, N. (2017) Mindfulness and Cognitive Functions: Toward a Unifying Neurocognitive Framework. Mindfulness, 8, 1-9. https://doi.org/10.1007/s12671-016-0654-1

[9] Mealer, M., Jones, J., Newman, J., McFann, K., Rothbaum, B. and Moss, M. (2012) The Presence of Resilience Is Associated with a Healthier Psychological Profile in Intensive Care Unit (ICU) Nurses: Results of a National Survey. International Journal of Nursing Studies, 49, 292-299. https://doi.org/10.1016/j.ijnurstu.2011.09.015

[10] Dimidjian, S. and Segal, Z.V. (2015) Prospects for a Clinical Science of Mindfulness-Based Intervention. American Psychologist, 70, 593-620.

https://doi.apa.org/doi/10.1037/a0039589

[11] Lau, M.A. and Segal, ZV. (2007) Mindfulness-Based Cognitive Therapy as a Relapse Prevention Approach to Depression. In: Witkiewitz, K.A. and Alan Marlatt, G., Eds., Therapist's Guide to Evidence-Based Relapse Prevention, Academic Press, Cambridge, 73-90. https://doi.org/10.1016/B978-012369429-4/50034-3

[12] Goyal, M., Singh, S., Sibinga, E.M., Gould, N.F., Rowland-Seymour, A., Sharma, R., Berger, Z., Sleicher, D., Maron, D.D., Shihab, H.M., Ranasinghe, P.D., Linn, S., Saha, S., Bass, E.B. and Haythornthwaite, J.A. (2014) Meditation Programs for Psychological Stress and Well-Being: A Systematic Review and Meta-Analysis. JAMA Internal Medicine, 174, 357-368.

https://doi.org/10.1001/jamainternmed.2013.13018

[13] Hofmann, S.G., Sawyer, A.T., Witt, A.A. and Oh, D. (2010) The Effect of Mindfulness-Based Therapy on Anxiety and Depression: A Meta-Analytic Review. Journal of Consulting and Clinical Psychology, 78, 169-183. https://doi.apa.org/doi/10.1037/a0018555

[14] Piet, J. and Hougaard, E. (2011) The Effect of Mindfulness-Based Cognitive Therapy for Prevention of Relapse in Recurrent Major Depressive Disorder: A Systematic Review and Meta-Analysis. Clinical Psychology Review, 31, 1032-1040. https://doi.org/10.1016/j.cpr.2011.05.002

[15] Dimidjian, S., Beck, A., Felder, J.N., Boggs, J.M., Gallop, R. and Segal, Z.V. (2014) Web-Based Mindfulness-Based Cognitive Therapy for Reducing Residual Depressive Symptoms: An Open Trial and Quasi-Experimental Comparison to Propensity Score Matched Controls. Behaviour Research and Therapy, 63, 83-89.

https://doi.org/10.1016/j.brat.2014.09.004 
[16] Maslach, C., Schaufeli, W.B. and Leiter, MP. (2001) Job Burnout. Annual Review of Psychology, 52, 397-422. https://doi.org/10.1146/annurev.psych.52.1.397

[17] Connor, K.M. and Davidson, J.R. (2003) Development of a New Resilience Scale: The Connor-Davidson Resilience Scale (CD-RISC). Depression and Anxiety, 18, 76-82. https://doi.org/10.1002/da.10113

[18] Foa, E.B. and Meadows, E.A. (1997) Psychosocial Treatments for Posttraumatic Stress Disorder: A Critical Review. Annual Review of Psychology, 48, 449-480. https://doi.org/10.1146/annurev.psych.48.1.449

[19] Mealer, M., Hodapp, R., Conrad, D., Dimidjian, S., Rothbaum, B.O. and Moss, M. (2017) Designing a Resilience Program for Critical Care Nurses. AACN Advanced Critical Care, 28, 359-365. https://doi.org/10.4037/aacnacc2017252

[20] Kalliath, T.J., O’Driscoll, M.P., Gillespie, D.F. and Bluedorn, A.C. (2000) A Test of the Maslach Burnout Inventory (MBI-HSS) in Three Samples of Healthcare Professionals. Work \& Stress, 14, 35-50. https://doi.org/10.1080/026783700417212

[21] Gil-Monte, P.R. (2005) Factorial Validity of the Maslach Burnout Inventory (MBIHSS) among Spanish Professionals. Revista de Saúde Pública, 39, 1-8. https://doi.org/10.1590/S0034-89102005000100001

[22] Maslach, C. (1979) Burned-Out. Can J Psychiatr Nurs, 20, 5-9.

[23] Mueser, K.T., Salyers, M.P., Rosenberg, S.D., Ford, J.D., Fox, L. and Carty, P. (2001) Psychometric Evaluation of Trauma and Posttraumatic Stress Disorder Assessments in Persons with Severe Mental Illness. Psychological Assessment, 13, 110-117. https://doi.apa.org/doi/10.1037/1040-3590.13.1.110

[24] Sheeran, T. and Zimmerman, M. (2002) Screening for Posttraumatic Stress Disorder in a General Psychiatric Outpatient Setting. Journal of Consulting and Clinical Psychology, 70, 961-966. https://doi.apa.org/doi/10.1037/0022-006X.70.4.961

[25] Aylard, P.R., Gooding, J.H., McKenna, P.J. and Snaith, R.P. (1987) A Validation Study of Three Anxiety and Depression Self-Assessment Scales. Journal of Psychosomatic Research, 31, 261-268. https://doi.org/10.1016/0022-3999(87)90083-3

[26] Bjelland, I., Dahl, A.A., Haug, T.T. and Neckelmann, D. (2002) The Validity of the Hospital Anxiety and Depression Scale. An Updated Literature Review. Journal of Psychosomatic Research, 52, 69-77. https://doi.org/10.1016/S0022-3999(01)00296-3

[27] Larsen, D., Attkisson, C., Hargreaves, W. and Nguyen, T. (1979) Assessment of Client/Patient Satisfaction: Development of a General Scale. Evaluation and Program Planning, 2, 197-207. https://doi.org/10.1016/0149-7189(79)90094-6

[28] Nguyen, T.D., Attkisson, C.C. and Stegner, B. (1983) Assessment of Patient Satisfaction: Development and Refinement of a Service Evaluation Questionnaire. Evaluation and Program Planning, 6, 299-313. https://doi.org/10.1016/0149-7189(83)90010-1

[29] Schneider, A., Forster, J. and Mealer, M. (2020) Exploratory and Confirmatory Factor Analysis of the Maslach Burnout Inventory to Measure Burnout Syndrome in Critical Care Nurses. Journal of Nursing Measurement, 28. 